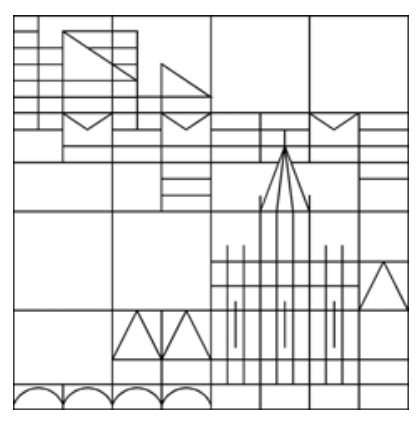

\title{
Formation of singularities in one-dimensional thermoelasticity with second sound
}

\author{
Yuxi Hu \\ Reinhard Racke
}

Konstanzer Schriften in Mathematik

Nr. 305, Juli 2012

ISSN 1430-3558

Konstanzer Online-Publikations-System (KOPS)

URL: http://nbn-resolving.de/urn:nbn:de:bsz:352-197104

(C) Fachbereich Mathematik und Statistik

Universität Konstanz

Fach D 197, 78457 Konstanz, Germany 



\title{
FORMATION OF SINGULARITIES IN ONE-DIMENSIONAL THERMOELASTICITY WITH SECOND SOUND
}

\author{
YUXI HU AND REINHARD RACKE
}

\begin{abstract}
We investigate the formation of singularities in thermoelasticity with second sound. Transforming into Euler coordinates and combining ideas from Sideris [14], used for compressible fluids, and Tarabek [15], used for small data large time existence in second sound models, we are able to show that there are in general no global smooth solutions for large initial data. In contrast to the situation for classical thermoelasticity, we require largeness of the data itself, not of its derivatives.
\end{abstract}

Keywords: singularities; thermoelasticity; second sound; compressible fluids; large data

AMS classification code: 35 L 60, 35 B 44

\section{INTRODUCTION}

We consider blow-up phenomena in one-dimensional nonlinear thermoelasticity with second sound. The - essentially nonlinear - equations are described as follows:

$$
\left\{\begin{array}{l}
\omega_{t}=v_{x}, \\
v_{t}=S_{x}, \\
e_{t}=S v_{x}-q_{x}, \\
\tau q_{t}+q+k \theta_{x}=0,
\end{array}\right.
$$

where $\omega, v, S, e, \eta$ denote strain, velocity, Piola-Kirchhoff stress tensor, internal energy, and entropy, respectively, and the constitutive equations are given by

$$
\psi=e-\theta \eta, \quad \psi_{\omega}=S, \quad \psi_{\theta}=-\eta,
$$

where $\psi=\psi(\omega, \theta, q)$ is the Helmholtz free energy, and $\theta$ and $q$ denote the temperature and the heat flux, respectively. The parameter $\tau$ is a positive constant, the relaxation constant. $k>0$ is also a given constant. For a derivation of the equations see [12] or [11], where we have taken Cattaneo's law $(1)_{4}$ in the linearized form; see also [7] for the limit case $\tau=0$.

We shall consider the Cauchy problem for the functions

$$
\omega, v, \theta, q: \mathbb{R} \times[0, \infty) \longrightarrow \mathbb{R}
$$

with initial conditions

$$
\left(\omega(x, 0), v(x, 0), \theta(x, 0), q(x, 0)=\left(\omega_{0}(x), v_{0}(x), \theta_{0}(x), q_{0}(x)\right),\right.
$$


where $x \in \mathbb{R} ; S(x, 0), e(x, 0), \eta(x, 0)$ can be determined through the constitutive equations (2).

For the limit case $\tau=0$, the equations (1) and (2) constitute the system of onedimensional classical thermoelasticity, in which the relation between the heat flux and the temperature is governed by Fourier's law,

$$
q=-k \theta_{x} .
$$

For this system, global existence of solutions for small data has been established by Hrusa and Tarabek in [6]. Later, the formation of singularities for large data was considered by Dafermos and Hsiao [1], Hrusa and Messaoudi [5], and Hansen [3]. They proved that if the derivatives of the initial data are sufficiently large (in appropriate norms), then the derivatives of the solution will necessarily tend to infinity in finite time.

In case $\tau>0$, Fourier's law is replaced by Cattaneo's law $(1)_{4}$ for heat conduction, and the corresponding system is called thermoelasticity with second sound reflecting the appearance of heat waves with finite propagation speed. In [15], Tarabek has established a global existence theorem for small initial data, and the decay to an equilibrium. The local existence theorem, stated in [15] with a hint to the paper of Hughes, Kato and Marsden [4], was completely proved, and the decay rates for global solutions were given by Racke and Wang [12]. It is a natural question to ask whether a global solution exists for any large data, or whether singularity will form in finite time if the data are sufficiently large.

Meanwhile, quite a number of results nourish the expectation that the dissipative effect induced by Fourier's law is not weaker (cp. [11] for a survey in thermoelasticity) but really stronger than the damping effect induced by Cattaneo's law, see [2, 9] for situations in bounded domains where one has exponential stability for Fourier's law but no exponential stability for Cattaneo's law. Therefore, one might expect that smooth solutions blow up in finite time for sufficiently large data, since this is known under the Fourier law, i.e. in classical thermoelasticity, as explained above. However, there seem to be no results yet about the formation of singularities for these system. We shall fill this gap.

The methods having been used for showing the blow-up results in classical thermoelasticity do not carry over to our situation due to the change of the character of the system from a hyperbolic-parabolic to a purely hyperbolic one. An appropriate transformation of variables, the choice of constitutive equations as model system, and choosing the right functionals for the blow-up arguments will allow us to obtain a blow-up result.

We will show that the maximal existence time for smooth solutions of system (1) with sufficiently large initial data must be finite. In contrast to [5], we require largeness of the solution (initially) itself but not of its derivatives. More precisely, we shall assume that the initial data have sufficiently large compact support, and the initial velocity should be large enough in a sense to be made precise later, see Theorem 4.1 . 
The method we use is mainly motivated by Sideris' paper [14], in which he showed several blow-up results for compressible fluid system, using finite propagation speed and appropriate averaged quantities. We shall first transform system (1) in order to get a compressible fluid -like system for which we can extend Sideris' approach. The system (1) will have the property of finite propagation speed, which is guaranteed by the hyperbolicity of the system (1). Introducing some proper averaged quantities, and using energy estimates derived from [15], we obtain the desired blow-up result.

So, the new ingredients of our paper are a first blow-up result in thermoelasticity with second sound, a short proof using Euler coordinates and corresponding elaborate averaged quantities and estimates.

The paper is organized as follows. In Section 2 we recall a local existence theorem and describe the chosen constitutive equations. In Section 3 we establish the finite propagation speed of the system and transform to Euler coordinates. Section 4 presents the main theorem (Theorem 4.1), and Section 5 its proof.

\section{LOCAL EXISTENCE AND CONSTITUTIVE EQUATIONS}

As in [15], we assume that the Helmholtz energy takes the following form:

$$
\psi(\omega, \theta, q)=\psi_{0}(\omega, \theta)+\frac{1}{2} \chi(\omega, \theta) q^{2},
$$

for some smooth function $\psi_{0}$ and with $\chi(\omega, \theta):=\frac{\tau(\omega, \theta)}{\theta \kappa(\omega, \theta)}$. The Clausius-Duhem inequality

$$
\psi_{t}+\eta \theta_{t}-S \omega_{t}+\frac{q \theta_{x}}{\theta} \leq 0
$$

holds under this special choice of the Helmholtz free energy. The strain and the temperature are required to satisfy

$$
\omega>-1, \quad \theta>0 .
$$

Moreover, it is reasonable to assume that there exists a fixed temperature $\bar{\theta}$ such that when $\omega=q=0$ and $\theta=\bar{\theta}$, the specific heat, the elastic modulus, the thermal relaxation time and the thermal conductivity are positive, while the stress-temperature modulus is non-vanishing, i.e.,

$$
e_{\theta}(0, \bar{\theta}, 0)>0, S_{\omega}(0, \bar{\theta}, 0)>0, \tau(0, \bar{\theta})>0, \kappa(0, \bar{\theta})>0, S_{\theta}(0, \bar{\theta}, 0) \neq 0 .
$$

We shall choose $\delta>0$ small enough such that $e_{\theta}, S_{\omega}, \tau, \kappa,\left|S_{\theta}\right|$ are positive and bounded away from zero on

$$
\Omega:=(-\delta, \delta) \times(\bar{\theta}-\delta, \bar{\theta}+\delta) \times(-\delta, \delta) .
$$

Now we present a local existence theorem for the problem $(1),(4)$ : (see $[4,12,15])$

Lemma 2.1. Suppose that (7) holds. Let $\omega_{0}, v_{0}, \theta_{0}, q_{0}: \mathbb{R} \rightarrow \mathbb{R}$ be given with

$$
\begin{gathered}
\omega_{0}, v_{0}, \theta_{0}, q_{0} \in H^{2}(\mathbb{R}), \\
\forall x \in \mathbb{R}:\left(\omega_{0}, \theta_{0}, q_{0}\right)(x) \in \Omega .
\end{gathered}
$$


Then the initial-value problem (1),(4) has a unique solution $(\omega, v, \theta, q)$ on a maximal time interval $\left[0, T_{0}\right)$, for some $T_{0}>0$, with

$$
(\omega, v, \theta-\bar{\theta}, q) \in C^{0}\left(\left[0, T_{0}\right), H^{2}(\mathbb{R})\right) \cap C^{1}\left(\left[0, T_{0}\right), H^{1}(\mathbb{R})\right),
$$

and

$$
\forall x \in \mathbb{R}, \forall t \in\left[0, T_{0}\right):(\omega(x, t), \theta(x, t), q(x, t)) \in \Omega .
$$

We consider special constitutive equations of the form:

$$
\left\{\begin{array}{l}
\psi=C_{v} \theta-\theta R \ln \left((\omega+1) \theta^{\frac{1}{\gamma-1}}\right)+\frac{\tau}{2 \kappa \theta} q^{2}, \\
S=-R \frac{\theta}{\omega+1}, \\
e=C_{v} \theta+\frac{\tau}{\kappa \theta} q^{2}, \\
\eta=R \ln (\omega+1)+C_{v} \ln \theta+\frac{\tau}{2 \kappa \theta^{2}} q^{2},
\end{array}\right.
$$

where $\tau, \kappa, R, C_{v}$ are positive constants, and

$$
\gamma:=1+\frac{R}{C_{v}}
$$

We choose $C_{v}$ large enough such that

$$
C_{v}>\frac{\tau \delta^{2}}{\kappa(\bar{\theta}-\delta)^{2}}
$$

For this special choice of the Helmholtz free energy $\psi$, we can easily check that the constitutive equations (2) hold, and that the conditions $S_{\omega}>0, \eta_{\theta}>0, S_{\theta} \neq 0$ are satisfied for $(\omega, \theta, q) \in \Omega$. Moreover, the Clausius-Duhem inequality (5) holds.

The idea for this special choice of the constitutive equations arises from the compressible fluid system for a polytropic gas. In order to use and to extend the methods from Sideris [14], we have to have an internal energy $e$ and a stress tensor $S$ to be related in a similar way as for a polytropic gas - except for the quadratic terms for heat flux arising here additionally.

The constitutive equations (12) satisfy the assumptions used to establish the global existence of smooth solutions (see [15]) when the data are sufficiently small. Moreover, they are fully compatible with the second law of thermodynamics; in particular there is a free energy.

\section{Finite propagation SPEed AND Euler COORDinAtes}

We shall first show the finite propagation speed property for system (1).

Lemma 3.1. Let $(\omega, v, \theta, q)$ be a local solution to (1),(4) on $\left[0, T_{0}\right)$. Let $M>0$. Assume that the initial data $\left(\omega_{0}, v_{0}, \theta_{0}-\bar{\theta}, q_{0}\right)$ are compactly supported in $(-M, M)$ and $\left(\omega_{0}, \theta_{0}, q_{0}\right) \in \Omega$. Then there exists a constant $\sigma$ such that

$$
(\omega(\cdot, t), v(\cdot, t), \theta(\cdot, t), q(\cdot, t))=(0,0, \bar{\theta}, 0)=:(\bar{\omega}, \bar{v}, \bar{\theta}, \bar{q})
$$

on $D(t):=\{x \in \mathbb{R}|| x \mid \geq M+\sigma t\}, \quad 0 \leq t<T_{0}$. 
Proof. We use an estimate on appropriately truncated cones, extending the standard arguments from $[10,13]$ to non-symmetric hyperbolic systems with lower-order terms.

With the constitutive equations (12) we rewrite (1) as

$$
A_{0}(U) U_{t}+A_{1}(U) U_{x}+B U=0,
$$

where $U:=(\omega, v, \theta, q)$ and

$$
\begin{gathered}
A_{0}(U):=\left(\begin{array}{cccc}
1 & 0 & 0 & 0 \\
0 & 1 & 0 & 0 \\
0 & 0 & C_{v}-\frac{\tau}{\kappa \theta^{2}} q^{2} & \frac{2 \tau}{\kappa \theta} q \\
0 & 0 & 0 & \tau
\end{array}\right), \\
A_{1}(U):=\left(\begin{array}{cccc}
0 & -1 & 0 & 0 \\
-\frac{R \theta}{(\omega+1)^{2}} & 0 & \frac{R}{\omega+1} & 0 \\
0 & \frac{R \theta}{\omega+1} & 0 & 1 \\
0 & 0 & \kappa & 0
\end{array}\right), B:=\left(\begin{array}{cccc}
0 & 0 & 0 & 0 \\
0 & 0 & 0 & 0 \\
0 & 0 & 0 & 0 \\
0 & 0 & 0 & 1
\end{array}\right) .
\end{gathered}
$$

Let $\bar{U}:=(\bar{\omega}, \bar{v}, \bar{\theta}, \bar{q})$ and $V:=U-\bar{U}$. Rewrite (15) as follows:

$$
V_{t}+A_{0}^{-1}(\bar{U}) A_{1}(\bar{U}) V_{x}+A_{0}^{-1}(\bar{U}) B V=H\left(\bar{U}, U, U_{t}, U_{x}\right),
$$

where

$$
H\left(\bar{U}, U, U_{t}, U_{x}\right):=A_{0}^{-1}(\bar{U})\left(A_{0}(\bar{U})-A_{0}(U)\right) U_{t}+A_{0}^{-1}(\bar{U})\left(A_{1}(\bar{U})-A_{1}(U)\right) U_{x} .
$$

The four different real eigenvalues

$$
\lambda_{1}<\lambda_{2}<0<\lambda_{3}<\lambda_{4}
$$

of $A_{0}^{-1}(\bar{U}) A_{1}(\bar{U})$ are easily computed to be

$$
\lambda_{1 / 2 / 3 / 4}= \pm \sqrt{\frac{1}{2}\left(\frac{\kappa}{\tau C_{v}}+\frac{R^{2} \bar{\theta}}{C_{v}}+R \bar{\theta} \pm \sqrt{\left(\frac{\kappa}{\tau C_{v}}+\frac{R^{2} \bar{\theta}}{C_{v}}+R \bar{\theta}\right)^{2}-\frac{4 R \bar{\theta} \kappa}{\tau C_{v}}}\right)}
$$

Therefore, there exists an invertible matrix $L(\bar{U})$ and a diagonal matrix $\Lambda(\bar{U})$ such that

$$
L(\bar{U}) A_{0}^{-1}(\bar{U}) A_{1}(\bar{U})=\Lambda(\bar{U}) L(\bar{U}) .
$$

Let $W:=L(\bar{U}) V$, then

where

$$
W_{t}+\Lambda(\bar{U}) W_{x}+\tilde{B} W=\tilde{H}
$$

$$
\tilde{B}:=L(\bar{U}) A_{0}^{-1}(\bar{U}) B L^{-1}(\bar{U}), \quad \tilde{H}:=L(\bar{U}) H .
$$

Now take $\sigma$ to be the largest eigenvalue of $A_{0}^{-1}(\bar{U}) A_{1}(\bar{U})$,

$$
\sigma:=\lambda_{4}
$$

Let $0 \leq t_{0}<T_{0}$, and let $x_{0} \in D\left(t_{0}\right)$. Define, for $0 \leq \tau \leq t_{0}$,

$$
P(\tau):=\left\{x|| x-x_{0} \mid \leq \sigma\left(t_{0}-\tau\right)\right\}
$$


and

$$
Q(\tau):=\{(x, t) \mid x \in P(t), 0 \leq t \leq \tau\} .
$$

The outer normal to $Q(\tau)$ along the lateral surface

$$
Q^{+}(\tau):=\left\{(x, t): x_{0}-x=\sigma\left(t_{0}-t\right), 0 \leq t \leq \tau\right\}
$$

is given by

$$
n^{+}:=\frac{(-1, \sigma)}{\sqrt{1+\sigma^{2}}}
$$

and along the lateral surface

$$
Q^{-}(\tau):=\left\{(x, t): x_{0}-x=-\sigma\left(t_{0}-t\right), 0 \leq t \leq \tau\right\}
$$

it is given by

$$
n^{-}:=\frac{(1, \sigma)}{\sqrt{1+\sigma^{2}}} .
$$

Now, multiplying (19) by $W$ and integrating over $Q(\tau),\left(0<\tau<t_{0}\right)$, using the divergence theorem, we obtain

$$
\begin{array}{r}
\int_{P(\tau)}|W|^{2} \mathrm{~d} x+\int_{Q^{+}(\tau)}(\sigma-\Lambda) W \cdot W \mathrm{~d}(x, t) \\
+\int_{Q^{-}(\tau)}(\sigma I+\Lambda) W \cdot W \mathrm{~d} x \mathrm{~d} t \leq C \int_{0}^{\tau} \int_{P(\tau)}|W|^{2} \mathrm{~d} x \mathrm{~d} t .
\end{array}
$$

Here $C$ denotes a positive constant (varying in the sequel), and we have used the fact that

$$
|\tilde{H} \cdot W| \leq C|W|^{2},
$$

which follows from the fact that $U, U_{t}, U_{x}$ are bounded on the domain of integration since $U \in C^{0}\left(\left[0, T_{0}\right), H^{2}(\mathbb{R})\right) \cap C^{1}\left(\left[0, T_{0}\right), H^{1}(\mathbb{R})\right)$.

By definition of $\sigma$, the second and third term on the left hand-side of (20) are positive. So, by Gronwall's inequality, we get $W=0$ on $P(\tau)$. Since $L(\bar{U})$ is invertible, $U(x, t)=\bar{U}$ on $P(\tau)$ for any $0<\tau<t_{0}$. Thus, $U\left(x_{0}, t_{0}\right)=\bar{U}$ and the proof is finished.

Now, we transform from Lagrange to Euler coordinates,

$$
\left\{\begin{array}{l}
\mathrm{d} y=(\omega+1) \mathrm{d} x+v \mathrm{~d} t, \\
\mathrm{~d} s=\mathrm{d} t .
\end{array}\right.
$$

Without confusion, we still denote $(y, s)$ by $(x, t)$. Then system $(1)$ becomes

$$
\left\{\begin{array}{l}
\rho_{t}+(\rho v)_{x}=0, \\
\rho\left(v_{t}+v \cdot v_{x}\right)=S_{x}, \\
\rho\left(e+\frac{v^{2}}{2}\right)_{t}+\rho v\left(e+\frac{v^{2}}{2}\right)_{x}=(S v-q)_{x}, \\
\rho \tau\left(q_{t}+v q_{x}\right)+\rho q+\kappa \theta_{x}=0,
\end{array}\right.
$$


where

$$
\rho:=\frac{1}{\omega+1} .
$$

We note that this transformation does not change the size of a given compact support of the solution.

\section{Statement of the Main theorem}

We define some useful averaged quantities:

$$
\begin{aligned}
G(t) & :=\int_{\mathbb{R}}(E(x, t)-\bar{E}) \mathrm{d} x, \\
F(t) & :=\int_{\mathbb{R}} x \rho v(x, t) \mathrm{d} x,
\end{aligned}
$$

where $E(x, t):=\rho\left(e+\frac{1}{2} v^{2}\right)$ is the total energy, $\bar{\rho}:=\frac{1}{\bar{\omega}+1}=1$, and $\bar{E}, \bar{S}, \bar{e}$ are defined through the constitutive equations (12), i.e.,

$$
\bar{E}:=\bar{e}:=C_{v} \bar{\theta}, \bar{S}:=-R \bar{\theta} .
$$

We mention that the functionals defined above exist since the solution $(\rho-1, v, \theta-$ $\bar{\theta}, q)$ are zero on the set $D(t)$ defined in Lemma 3.1.

Our main result is stated as follows:

Theorem 4.1. We assume that the initial data satisfy the assumptions in Lemma 3.1. Moveover, we assume that

$$
G(0)>0
$$

Then there exists $v_{0}$ satisfying

$$
F(0)>\frac{16 \sigma \max \rho_{0}}{3-\gamma} M^{2}, \quad \text { with } \quad 1<\gamma<3,
$$

such that the length $T_{0}$ of the maximal interval of existence of a smooth solution $(\omega, v, \theta, q)$ of $(1),(4)$ is finite, provided the compact support of the initial data is sufficiently large.

Remark 4.1. It is easy to show that there exist initial data $\left(\rho_{0}, v_{0}, \theta_{0}, q_{0}\right)$ such that (23) holds. For example, we can take $\theta_{0} \geq \bar{\theta}$ appropriately, then (23) follows immediately.

Remark 4.2. In [15], Tarabek has shown that a unique global solution exists provided that the initial data are small enough, i.e.,

$$
\left\|\left(\omega_{0}, v_{0}, \theta_{0}, q_{0}\right)\right\|_{H^{2}} \leq \varepsilon,
$$

for some small $\varepsilon>0$. Our theorem shows that a global smooth solution can not exist provided the initial velocity $v_{0}$ is large in the sense of (24).

Compared to the blow-up result for the Euler system in [14], we require that the size of the compact support of initial data should be large. We do not need any conditions on thte initial mass. This is due to the different structure of the two systems. 
It is interesting to observe that the blow-up results in classical thermoelasticity $(\tau=0)$ are different (see [5]). This is due to the different structures of these two system, i.e., one is hyperbolic-parabolic, and one is purely hyperbolic. There is no uniform method for these systems.

\section{Proof of the MAIN THEOREM}

Now we are ready to prove Theorem 4.1. First we can easily get from $(22)_{3}$ that $G$ is constant,

$$
G(t)=G(0)
$$

We have

$$
F^{\prime}(t)=\int\left[\rho|v|^{2}+(\bar{S}-S)\right] \mathrm{d} x
$$

Then, by the constitutive equations (12) and using (25), we get

$$
\begin{aligned}
\int_{B_{t}} S \mathrm{~d} x & =-\int_{B_{t}} R \rho\left(\frac{e}{C_{v}}-\frac{\tau}{\kappa C_{v} \theta} q^{2}\right) \mathrm{d} x \\
& =\int_{B_{t}} \frac{R \rho \tau}{\kappa C_{v} \theta} q^{2} \mathrm{~d} x-\int_{B_{t}} \frac{R}{C_{v}}\left(E-\frac{1}{2} \rho v^{2}\right) \mathrm{d} x \\
& \leq \int_{B_{t}}\left(\frac{R \rho \tau}{\kappa C_{v} \theta} q^{2}+\frac{R \rho}{2 C_{v}} v^{2}\right) \mathrm{d} x-\int_{B_{t}} \frac{R}{C_{v}} \bar{E} \\
& =\int_{B_{t}}\left(\frac{R \rho \tau}{\kappa C_{v} \theta} q^{2}+\frac{R \rho}{2 C_{v}} v^{2}\right) \mathrm{d} x+\int_{B_{t}} \bar{S} \mathrm{~d} x,
\end{aligned}
$$

where $B_{t}=\{(x, t) ;|x| \leq M+\sigma t\}$. Therefore,

$$
\int_{B_{t}}(\bar{S}-S) \mathrm{d} x \geq-\int_{B_{t}} \frac{\gamma-1}{2} \rho v^{2} \mathrm{~d} x-\int_{B_{t}} \frac{(\gamma-1) \rho \tau}{\kappa \theta} q^{2} \mathrm{~d} x
$$

Thus, (26) turns into

$$
F^{\prime}(t) \geq\left(1-\frac{\gamma-1}{2}\right) \int_{B_{t}} \rho v^{2} \mathrm{~d} x-\int_{B_{t}} \frac{(\gamma-1) \tau}{\kappa \theta} \rho q^{2} \mathrm{~d} x
$$


By the definition of $F(t)$, we know

$$
\begin{aligned}
F(t) & =\int_{\mathbb{R}} x \rho(x, t) v(x, t) \mathrm{d} x \\
& \leq\left(\int_{B_{t}} x^{2} \rho \mathrm{d} x\right)^{\frac{1}{2}}\left(\int_{B_{t}} \rho v^{2} \mathrm{~d} x\right)^{\frac{1}{2}} \\
& \leq(M+\sigma t)\left(\int_{B_{t}} \rho \mathrm{d} x\right)^{\frac{1}{2}}\left(\int_{B_{t}} \rho v^{2} \mathrm{~d} x\right)^{\frac{1}{2}} \\
& =(M+\sigma t)\left(\int_{B_{t}} \rho_{0} \mathrm{~d} x\right)^{\frac{1}{2}}\left(\int_{B_{t}} \rho v^{2} \mathrm{~d} x\right)^{\frac{1}{2}} \\
& \leq\left(\max \rho_{0}\right)^{\frac{1}{2}}(M+\sigma t)(2(M+\sigma t))^{\frac{1}{2}}\left(\int_{B_{t}} \rho v^{2} \mathrm{~d} x\right)^{\frac{1}{2}} .
\end{aligned}
$$

In the following we assume that

$$
F(t) \geq c_{1}>0
$$

holds for some constant $c_{1}$, and for any $t$, which will be justified below. Then we get

$$
\int_{B_{t}} \rho v^{2} \mathrm{~d} x \geq\left(2 \max \rho_{0}(M+\sigma t)^{3}\right)^{-1} F^{2} .
$$

Therefore, equation (27) becomes

$$
F^{\prime}(t) \geq \frac{3-\gamma}{2}\left(2 \max \rho_{0}(M+\sigma t)^{3}\right)^{-1} F^{2}-\frac{2(\gamma-1) \tau}{\kappa \bar{\theta}} \int_{\mathbb{R}} \rho q^{2} \mathrm{~d} x,
$$

where we used a priori estimate for $\theta$, i.e., $\theta>\bar{\theta}-\delta>\frac{\bar{\theta}}{2}$. By exploiting an idea from Tarabek [15], we get the following relation:

$$
\partial_{t}\left(\psi-\tilde{\psi}-\tilde{S} \omega+(\theta-\bar{\theta}) \eta+\frac{1}{2} v^{2}\right)+\frac{\bar{\theta}}{\kappa \theta^{2}} q^{2}=\partial_{x}\left((S-\tilde{S}) v-(\theta-\bar{\theta}) \frac{q}{\theta}\right)
$$

holds where $\tilde{S}=S(0, \bar{\theta}, 0), \tilde{\psi}=\psi(0, \bar{\theta}, 0)$. We use the transformation (21) in this equation and thus get the following energy estimates:

$$
\int_{\mathbb{R}} \rho\left(\omega^{2}+v^{2}+(\theta-\bar{\theta})^{2}+q^{2}\right) \mathrm{d} x+\int_{0}^{t} \int_{\mathbb{R}} \rho q^{2} \mathrm{~d} x \mathrm{~d} t \leq \int_{\mathbb{R}} \rho_{0}\left(\omega_{0}^{2}+v_{0}^{2}+\left(\theta_{0}-\bar{\theta}\right)^{2}+q_{0}^{2}\right) \mathrm{d} x .
$$

Then, dividing equation (30) by $F^{2}$ and integrating over $(0, t)$, we get

$$
\begin{aligned}
\frac{1}{F_{0}}-\frac{1}{F} & \geq-\frac{c_{3}}{2 c_{2}}\left(1+c_{2} t\right)^{-2}+\frac{c_{3}}{2 c_{2}}-\frac{2(\gamma-1) \tau}{\kappa \bar{\theta} c_{1}^{2}} \int_{\mathbb{R}} \rho_{0}\left(\omega_{0}^{2}+v_{0}^{2}+\left(\theta_{0}-\bar{\theta}\right)^{2}+q_{0}^{2}\right) \mathrm{d} x \\
& \geq-\frac{c_{3}}{2 c_{2}}\left(1+c_{2} t\right)^{-2}+\frac{c_{3}}{2 c_{2}}-c_{4}-c_{5}\left\|v_{0}\right\|_{L^{2}}^{2},
\end{aligned}
$$

where

$$
c_{2}:=\frac{\sigma}{M}, \quad c_{3}:=\frac{3-\gamma}{4 \max \rho_{0} M^{3}},
$$




$$
c_{4}:=\frac{12(\gamma-1) \delta^{2} M \max \rho_{0} \tau}{\bar{\theta} \kappa c_{1}^{2}}, \quad c_{5}:=\frac{2(\gamma-1) \max \rho_{0} \tau}{\kappa \bar{\theta} c_{1}^{2}} .
$$

We claim that there exists some initial value $v_{0}$ such that, for some large $M$, the following two conditions hold:

$$
\begin{gathered}
F_{0}>\frac{4 c_{2}}{c_{3}}, \\
\frac{c_{3}}{4 c_{2}} \geq c_{4}+c_{5}\left\|v_{0}\right\|_{L^{2}}^{2} .
\end{gathered}
$$

We shall prove this claim at the end of this section. We first can now justify the positivity of $F$ assumed in (28). Let

$$
c_{1}:=\frac{c_{2}}{c_{3}}
$$

From (34) we conclude that there is $t_{1}>0$ such that

$$
F(t) \geq c_{1} \quad \text { for } t \in\left[0, t_{1}\right] .
$$

If

$$
T^{*}:=\sup \left\{0<t_{1}<T_{0} \mid F \geq c_{1} \text { on }\left[0, t_{1}\right]\right\},
$$

we have the maximality of $T^{*}$, i.e. $T^{*}=T_{0}$, since the assumption that $T^{*}<T_{0}$ would yield by (33)

$$
\frac{1}{F} \leq \frac{1}{F_{0}}+c_{4}+c_{5}\left\|v_{0}\right\|_{L^{2}}^{2}
$$

and thus by (35)

$$
F \geq 2 c_{1} \quad \text { on }\left[0, T^{*}\right]
$$

contradicting the definition of $T^{*}$ as supremum.

Now, from (28), (33), and (35), we get for any $t \in\left[0, T_{0}\right)$,

$$
\frac{1}{F_{0}} \geq \frac{1}{F_{0}}-\frac{1}{F} \geq \frac{c_{3}}{4 c_{2}}-\frac{c_{3}}{2 c_{2}}\left(1+c_{2} t\right)^{-2} .
$$

Therefore, $T_{0}$ can not become arbitrarily large without contradicting (34).

Thus, the proof will be finished if we can show that (34),(35) hold for some admissible initial data $v_{0}$. We choose $v_{0} \in H^{2}(\mathbb{R}) \cap C^{1}(\mathbb{R})$ as follows:

$$
v_{0}(x):=\left\{\begin{array}{lc}
0, & x \in(-\infty,-M], \\
\frac{L}{2} \cos (\pi(x+M))-\frac{L}{2} & x \in(-M,-M+1], \\
-L, & x \in(-M+1,-1), \\
L \cos \left(\frac{\pi}{2}(x-1)\right), & x \in(-1,1], \\
L, & x \in(1, M-1], \\
\frac{L}{2} \cos (\pi(x-M+1))+\frac{L}{2} & x \in(M-1, M], \\
0, & x \in(M, \infty),
\end{array}\right.
$$


where $L$ is a positive constant to be determined later. We assume $M \geq 4$. Since

$$
F_{0}=\int \rho_{0} x v_{0} \mathrm{~d} x \geq \frac{L}{2} \min \rho_{0} M^{2},
$$

we can choose $L$ large enough, and independent of $M$, such that

$$
\frac{L}{2} \min \rho_{0}>\frac{4 \sigma \max \rho_{0}}{3-\gamma}
$$

Therefore, (34) holds. On the other hand, since $\left\|v_{0}\right\|_{L^{2}}^{2} \leq 2 L^{2} M$, we can choose $M$ sufficiently large such that

$$
2 L^{2} M \leq \frac{\kappa \bar{\theta} \sigma}{2(\gamma-1)(3-\gamma) \tau} M^{2}-6 \delta^{2} M
$$

Therefore, (35) holds too, and the proof of Theorem 4.1 is finished.

\section{REFERENCES}

[1] C.M. Dafermos, L. Hsiao, Development of singularities in solutions of the equations of nonlinear thermoelasticity, Quart. Appl. Math. 44 (1986), 463-474.

[2] H.D. Fernández Sare, R. Racke, On the stability of damped Timoshenko systems — Cattaneo versus Fourier law, Arch. Rational Anal. Mech. 194 (2009), 221-251.

[3] I. Hansen, Lebensdauer von klassischen Lösungen nichtlinearer Thermoelastizitätsgleichungen. Diploma thesis, University of Bonn (1994).

[4] T.J.R. Hughes, T. Kato, J.E. Marsden, well-posed quasi-linear second-order hyperbolic systems with applications to nonlinear elastodynamics and general relaticity, Arch. Rational Mech. Anal. 63(1977), 273-294.

[5] W.J. Hrusa, S.A. Messaoudi, On formation of singularities in one-dimensional nonlinear thermoelasticity, Arch. Rational Mech. Anal. 111 (1990), 135-151.

[6] W.J. Hrusa, M.A. Tarabek, On smooth solutions of the Cauchy problem in one-dimensional nonlinear thermoelasticity, Quart. Appl. Math. 47 (1989), 631-644.

[7] S. Jiang, R. Racke, Evolution equations in thermoelasticity, Chapman and Hall/CRC Monographs and Surveys in Pure and Appl. Math. Vol. 112, (2000).

[8] S. Kawashima, Systems of a hyperbolic-parabolic composite type, with applications to the equations of magnetohydrodynamics, Thesis, Kyoto University (1983).

[9] R. Quintanilla, R. Racke, Addendum to: Qualitative aspects of solutions in resonators, Arch. Mech. 63 (2011), 429-435.

[10] R. Racke, Lectures on nonlinear evolution equations. Initial value problems. Aspects of Mathematics E19. Friedr. Vieweg \& Sohn, Braunschweig/Wiesbaden (1992).

[11] R. Racke, Thermoelasticity, Handbook of Differential Equations, Chapter 4, C.M. Dafermos, M. Pokorný (eds.). Elsevier (2009), 315-386

[12] R. Racke, Y.G. Wang, Nonlinear well-posedness and rates of decay in thermoelasticity with second sound, J. Hyperbolic Differential Equations 5 (2008), 25-43.

[13] T.C. Sideris, Formation of singularities of solutions to nonlinear hyperbolic equations, Arch Rational Mech. Anal. 86 (1984), 369-381.

[14] T.C. Sideris, Formation of singularities in three-dimensional compressible fluids, Commun. Math. Phys. 101 (1985), 475-485.

[15] M.A. Tarabek, On the existence of smooth solutions in one-dimensional nonlinear thermoelasticity with second sound, Quart. Appl. Math. 50 (1992) 727-742. 
Yuxi Hu, Department of Mathematics, Shanghai Jiao Tong University, Shanghai 200240, P.R. China, huyuxi@sjtu.edu.cn

Reinhard Racke, Department of Mathematics and Statistics, University of Konstanz, 78457 Konstanz, Germany, reinhard.racke@uni-konstanz.de 